\title{
Synthesis of Asymptotically Stable Motion of a Robot Arm Manipulator
}

\begin{abstract}
S. P. Bezglasnyi ${ }^{1}$, E. S. Batina ${ }^{1}$, A. S. Vorobyov ${ }^{2}$
${ }^{1}$ S. P. Korolyov Samara State Aerospace University, Russia, 443086, Samara, Moskovskoe sh., 34 bezglasnsp@ rambler.ru, katja4-2@mail.ru

${ }^{2}$ State Research and Production Space-Rocket Center «TsSKB-Progress», Russia, 443009, Samara, Zemets str., 18, svirex.hide@gmail.com

The paper is about an active control problem. It solves the inverse problem of dynamics and concerns with construction of program motions of non-autonomous mechanical systems. This study is important and necessary in software design of automated systems for control of mechanisms. In particular, it is used in various modeling problems of robot-manipulators. Here, we construct all possible asymptotically stable program motions for a model of robots arm-manipulator, which is simulated by a mechanical system with three degrees of freedom. The control force is obtained in the form of closed form solution in the class of continuous functions. The stabilization problem is solved by the direct Lyapunov's method with the use of limiting functions and systems. In this case, we are able to restrict ourselves to Lyapunov's functions having constant sign derivatives. Our results are a valuable contribution to development of control mechanisms in robotics and engineering.
\end{abstract}

Key words: programm motion, stabilizing control, the method of Lyapunov's functions.

\section{References}

1. Afanasyev V. N., Kolmanovskii V. B., Nosov V. R. Matematicheskaia teoriia konstruirovaniia sistem upravleniia [The mathematical theory of design of control systems]. Moscow, Vyssh. shk., 1989, 447 p. (in Russian).

2. Letov A. M. Dinamika poleta $i$ upravleniia [Flight Dynamics and Control]. Moscow, Nauka, 1969, 359 p. (in Russian).

3. Galiullin A. S., Mukhametzyanov I. A., Mukharlyamov R. G., Furasov V. D. Postroenie sistem programmnogo dvizheniia [Building Systems software movement]. Moscow, Nauka, 1971, 352 p. (in Russian).

4. Zubov V. I. Problema ustoichivosti protsessov upravleniia [Stability problem management processes]. Leningrad, Shipbuilding, 1980, 375 p. (in Russian).

5. Smirnov E. Y., Pavlikov I. J., Shcherbakov P. P., Jurkov A. V. Upravlenie dvizheniem mekhanicheskikh sistem [Motion control of mechanical systems]. Lenin-

grad, Leningrad State University, 1985. 347 p. (in Russian).

6. Rush N., Abets P., Laloy M. Priamoi metod Liapunova $v$ teorii ustoichivost [Direct method of Lyapunov stability theory]. Moscow, Mir, 1980, 301 p. (in Russian).

7. Artstein Z. Topological dynamics of an ordinary equations. J. Differ. Equat., 1977, vol. 23, pp. 216-223.

8. Andreev A. S. The asymptotic stability and instability of the zeroth solution of a non- autonomous system. J. Appl. Math. Mech., 1984, vol. 48, no. 2, pp. 225-232. (in Russian)

9. Bezglasnyi S. P. The stabilization of program motions of controlled nolinear mechanical systems. Korean $J$. Comput. and Appl. Math., 2004, vol. 14, no. 1-2, pp. 251266.

10. Bezglasnyi S. P., Mysina O. A. Stabilization of program motions of a rigid body on a moving platform. Izv. Sarat. Univ. N.S. Ser. Math. Mech. Inform., 2008, vol. 8, iss. 4, pp. 44-52 (in Russian).

\section{КОНЕЧНО-ЭЛЕМЕНТНЫЙ АНАЛИЗ ВЛИЯНИЯ КОНСТРУКЦИИ ОРТОДОНТИЧЕСКОГО АППАРАТА НА РАСШИРЕНИЕ ВЕРХНЕЙ ЧЕЛЮСТИ}

\author{
С. М. Босяков ${ }^{1}$, А. Н. Доста ${ }^{2}$, А. В. Винокурова ${ }^{3}$ \\ ${ }^{1}$ Кандидат фризико-математических наук, доцент касредры теоретической и прикладной механики, Белорусский государ- \\ ственный университет, Минск, bosiakov@bsu.by \\ ${ }^{2}$ Кандидат медицинских наук, доцент кафредры ортопедической стоматологии, Белорусский государственный медицинский \\ университет, Минск, dostastom75@mail.ru \\ 3 Аспирант кафедры теоретической и прикладной механики, Белорусский государственный университет, Минск, \\ janeraven@mail.ru \\ В работе представлены результаты конечно-элементного расчета напряженно-десрормированного состояния верхнече- \\ люстного комплекса человека, возникающего при активации ортодонтического аппарата HYRAX. Модели черепа и опорных \\ зубов верхнего зубного ряда получены на основании томограсрических данных для сухого интактного черепа взросло- \\ го человека. Конструкции ортодонтического аппарата отличаются расположением винта и стержней относительно неба. \\ Определены эквивалентные напряжения и перемещения костей верхней челюсти и опорных зубов. Показано, что при \\ расположении стержней и винта ортодонтического аппарата в горизонтальной плоскости в верхнечелюстном комплексе
}


возникают наиболее высокие напряжения, но наблюдается наклонно-вращательное перемещение зубов и перемещение вверх костей верхней челюсти. При активации ортодонтического аппарата с винтом, расположенным у неба, возникают нежелательные напряжения в костях черепа; перемещения костей и опорных зубов направлены вниз. Определены положения винта ортодонтического аппарата, обуславливающие поступательное перемещение костей верхней челюсти.

Ключевые слова: быстрое верхнечелюстное расширение, ортодонтический аппарат HYRAX, конечно-элементный анализ, напряженно-десрормированное состояние, черепно-лицевой комплекс.

\section{ВВЕДЕНИЕ}

Большинство методик коррекции сужений верхней челюсти применяют поперечные силы, которые развивают съемные или несъемные ортодонтические аппараты [1]. Технология верхнечелюстного расширения имеет долгую историю, развитие которой можно проследить по работам [2-5]. Влияние быстрого расширения верхней челюсти у пациентов без расщелины неба достаточно хорошо исследовано клинически [6-10] с использованием фотоупругости и голографии $[1,11-14]$. Реакция костей черепа на верхнечелюстное расширение исследована цефалометрически [15], а также посредством тензометрических измерений $[16,17]$. Для лучшего понимания влияния быстрого расширения неба выполнено достаточно много конечно-элементных исследований [2-5, 18-31]. В указанных клинических, экспериментальных и теоретических исследованиях показано, что использование ортодонтических аппаратов, развивающих трансверсальные силы, позволяет увеличить верхнечелюстной поперечный размер, но является достаточно сложной процедурой, вызывающей значительные побочные эффекты, в частности вывих опорных зубов и связанную с этим фенестрацию кортикальной пластины, резорбцию корня и рецессию десны [31]. Расширение верхней челюсти может ассоциироваться с ощущениями давления в различных областях ее сочленения, в частности, в области скуловых костей и переносицы, а также может сопровождаться рядом осложнений [32]. Для прогнозирования изменений в костях и хрящевых соединениях верхнечелюстного комплекса, а также представления о конечном терапевтическом эффекте в первую очередь необходимы сведения о нагрузке, развиваемой ортодонтическим аппаратом $[2,3,5,18,22,23,27,28,30]$. В то же время в большинстве конечно-элементных исследований, связанных с расчетом напряженно-деформированного состояния черепа или верхней челюсти при быстром верхнечелюстном расширении, не рассматривается реальная конструкция ортодонтического аппарата. Нагружение осуществляется за счет приложения сосредоточенных трансверсальных сил (от 1 Н до $300 \mathrm{H}$ ) к зубам, в частности, премолярам и молярам [2, $4,21-23,27]$, или посредством перемещений зубов на расстояния, соответствующие определенным количествам оборотов ортодонтического винта (от 0.2 мм до 5 мм) [18, 22, 24, 28, 30]. В работе [3] выполняется моделирование ортодонтического аппарата типа HYRAX, но, тем не менее, нагружение зубов осуществляется перемещением группы зубов верхнего зубного ряда, а не активацией винта аппарата. Важные результаты в этом направлении получены в исследовании [5], где, помимо высокоточной модели черепа человека, выполнено построение модели ортодонтического аппарата, и нагружение осуществляется как при смещении пружин аппарата, так и посредством приложения сосредоточенной нагрузки. После проведения сравнительного анализа авторами сделан вывод о том, что простые подходы, основанные на приложении сосредоточенной силы или перемещения, не отражают реальной ситуации и дают некачественые результаты при моделировании верхнечелюстного расширения. В настящей работе проведен анализ влияния конструкции ортодонтического аппарата HYRAX на возникающее после его начальной активации напряженно-деформированное состояние верхнечелюстного комплекса.

\section{2. МОДЕЛИРОВАНИЕ ЧЕРЕПА И ОРТОДОНТИЧЕСКОГО АППАРАТА}

Стереолитографическая модель черепа получена с применением программы для обработки медицинских изображений MIMICS 14.12 (Materialise's Interactive Medical Image Control Systems, Materialise BV, Leuven, Belgium) на основании 210 томогрофических изображений высушенного трупного интактного черепа взрослого человека с хорошо сохранившимися альвеолярными отростками и зубами. Отметим, что использование для томографии сухого черепа взрослого человека представляется достаточным для разработки твердотельной трехмерной геометрической модели $[2,21,22,24]$, тем более, что согласно исследованию [13] при действии ортодонтических сил на сухой череп начальная реакция аналогична реакции черепа в естественных условиях. Шаг томографических срезов составляет 1 мм. 
При генерации стереолитографической (STL) модели удалены первые и вторые премоляры верхней челюсти (14, 15 и 24, 25 зубы), а также постоянные моляры (16 и 26 зубы), на которые устанавливается ортодонтический аппарат. Конечно-элементная модель получена после обработки STL-модели в модуле 3-matic 6.1 пакета MIMICS. Импортирование дискретной модели черепа в программную среду пакета ANSYS Workbench 13 (ANSYS Inc., USA) выполнено с помощью компонента Finite Element Modeler. Полученная модель содержит 26445 узлов и 91731 элемент типа Solid72. Конечно-элементное разбиение выполнялось в автоматическом режиме. Результат импортирования и STL-модель показаны на рис. 1, $а$, б.

Моделирование периодонтальной связки не выполнялось, поскольку согласно работе [34] наличие периодонта практически не влияет на распределение напряжений в костях челюстно-лицевого комплекса при действии различных типов нагрузок. Шовные соединения верхнечелюстного комплекса также не учитывались в конечно-элементной модели. Это обусловлено тем, что в черепе взрослого человека швы частично или полностью окостеневают [18].

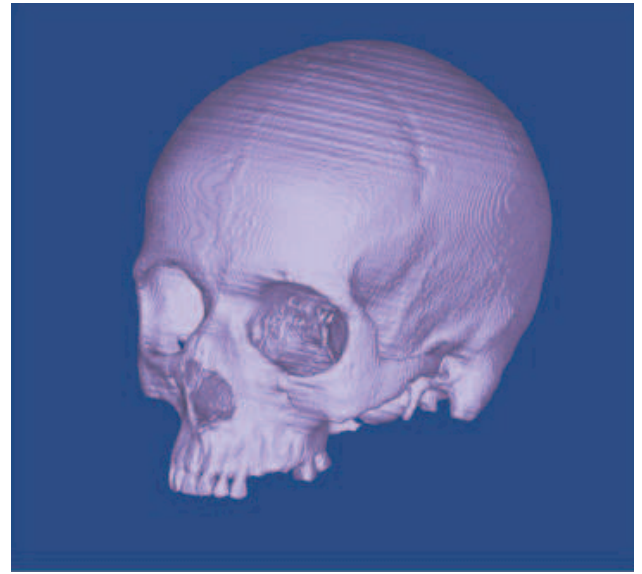

$a$

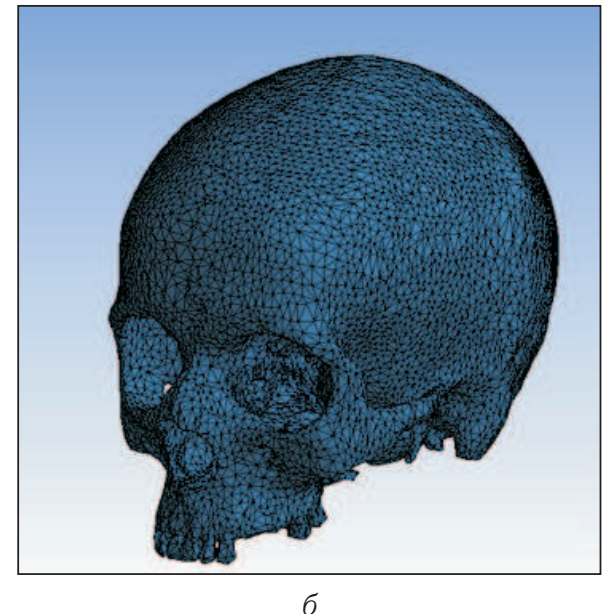

$\sigma$

Рис. 1. Стереолитографическая $(a)$ и конечно-элементная (б) модели интактного черепа человека без нижней челюсти

Твердотельные модели премоляров и первого моляра черепа человека также получены на основании томографических данных черепа человека с применением CAD-пакета SolidWorks 2010 (SolidWorks Corporation, USA). C помощью графических примитивов этого пакета построена модель ортодонтического аппарата HYRAX с коронками, устанавливаемыми на премоляры (14 и 24 зубы) и постоянные моляры (16 и 26 зубы), а также стержнями, соединяющими коронки и действующими на премоляры (15 и 25 зубы). Отметим, что в ортодонтическом аппарате HYRAX, используемом для раскрытия небного шва, активная часть (винт) фиксируется к пассивной (коронке или кольцу) посредством проволочных элементов, припаянных к небной поверхности колец или коронок, укрепляемых цементом на моляры и премоляры [1]. Реальная конструкция ортодонтического аппарата и его модель в пакете SolidWorks представлены на рис. $2, a$, б.

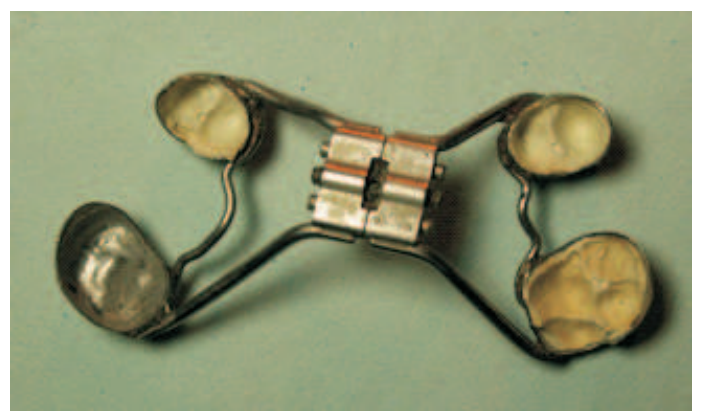

$a$

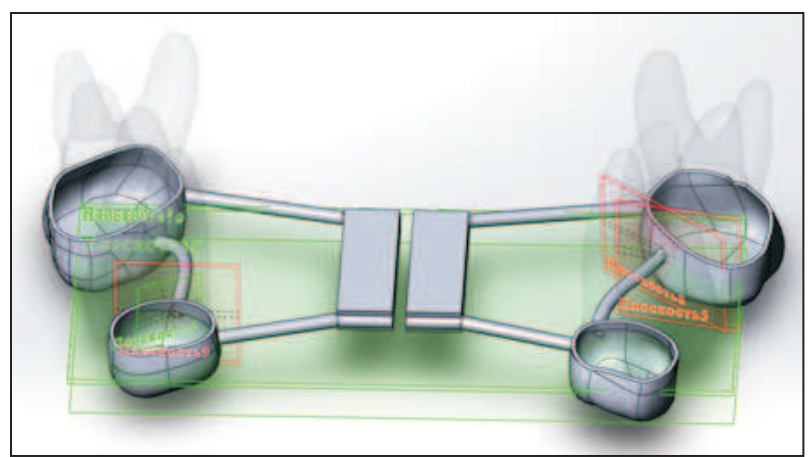

б

Рис. 2. Ортодонтический аппарат $(a)$ и его геометрическая модель (б), установленная на четвертые премоляры и постоянные моляры 
Длина и ширина пластинок модели ортодонтического аппарата, представленного на рис. 2, б, составляют 10 и 4 мм соответственно; радиус поперечного сечения стержней равен 1 мм, длина большего стержня составляет 11.05 мм, меньшего стержня -8.15 мм, толщина коронок -0.2 мм. Генерация конечно-элементной модели ортодонтического аппарата, премоляров и первого моляра выполнена с применением компонента Mechanical Model пакета Ansys Workbench 13. Общее количество элементов и узлов составляет 13320 и 26375 соответственно; максимальный размер полученных элементов для аппарата равен 1 мм, тип элемента - Mesh200. Модель аппарата и зубов после импортирования в модуль Finite Element Modeler добавлена в конечно-элементную модель черепа. При этом контакт между коронками ортодонтического аппарата и зубами верхней челюсти задан с помощью контактных элементов CONTA174 и TARGE170. Контакт между черепом и зубами задан с помощью контактных элементов CONTA173 и TARGE170. При описании контактного взаимодействия между коронками и зубами, а также между верхней челюстью и зубами выбран тип Bonded, соответствующий отсутствию скольжения и проникновения. Также отметим, что все щели между контактирующими поверхностями при таком типе контакта закрыты, то есть выполняется «склеивание» поверхностей.

Граничные условия, накладываемые на череп, соответствовали жесткой заделке узлов, находящихся в окрестности большого затылочного отверстия $[2,3,5]$. Перемещение каждой пластинки составляет 0.25 мм (соответствует активации винта ортодонтического аппарата на полоборота $[4,27]$ ) и направлено только трансверсально (в направлении оси $0 x$ ). Конечно-элементная модель верхнечелюстного комплекса с установленным ортодонтическим аппаратом и накладываемые граничные условия показаны на рис. $3, a$, б.

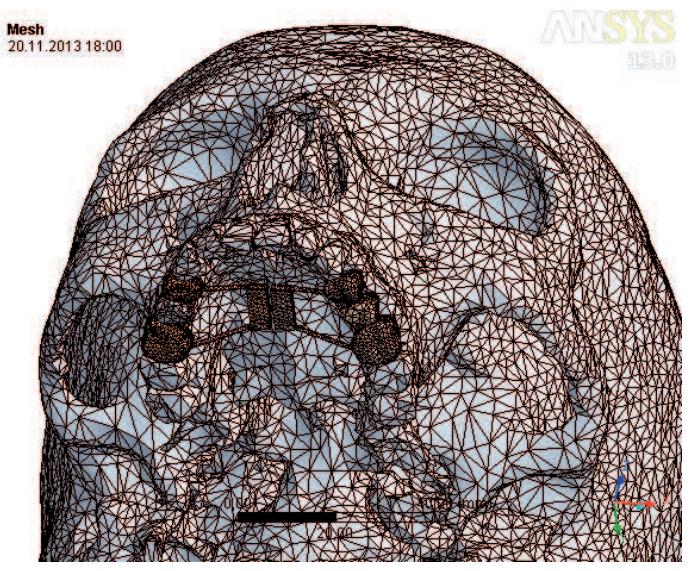

a

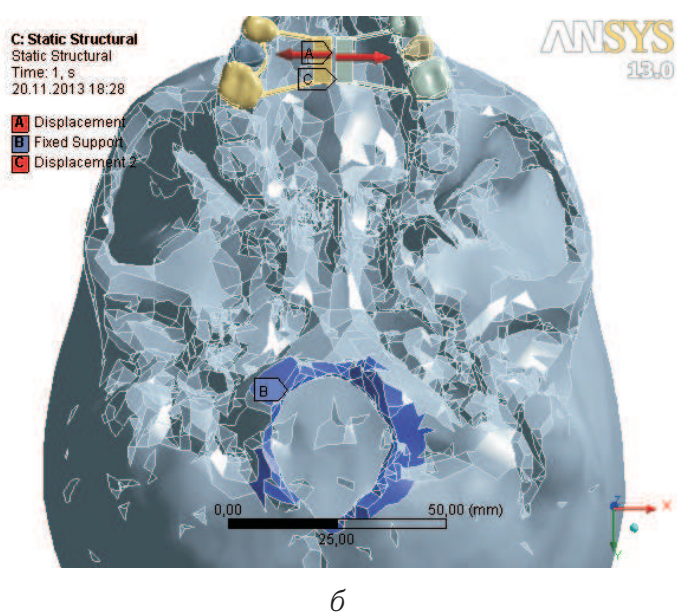

б

Рис. 3. Конечно-элементная модель верхней челюсти с установленным ортодонтическим аппаратом $(a)$ и граничные условия, накладываемые на конечно-элементную модель (б); граничные условия, отмеченные маркерами А и С, соответствуют перемещению пластинок ортодонтического аппарата в трансверсальном направлении (вдоль оси $0 x$ ); граничное условие, отмеченное маркером В соответствует жесткому закреп-

лению узлов черепа в окрестности большого затылочного отверстия

Конечно-элементный расчет напряженно-деформированного состояния черепа с установленным ортодонтическим аппаратом проводился для случая, когда модуль упругости материала, из которого изготовлены пластинка и стержни аппарата, составлял $E=200$ ГПа, коэффициент Пуассона $\nu=0.3$. Модуль упругости компактной (кортикальной) костной ткани и зубов равен $E=15$ ГПа и $E=20$ ГПа соответственно, коэффициент Пуассона как для кортикальной костной ткани, так и для зубов $\nu=0.3$ [28]. Рассматривались конструкция ортодонтического аппарата с расположением стержней и винта в горизонтальной плоскости, а также конструкции, в которых винт аппарата смещен ближе к небу относительно горизонтальной плоскости на $0.5,1,2$ и 8 мм. Геометрические размеры ортодонтических аппаратов остаются прежними, за исключением длин стержней, соединяющих винт аппарата с премолярами и винт с молярами. Для первого случая длина стержня изменяется от 8.15 до 12.20 мм, во втором случае длина изменяется от 11.05 до 16.45 мм.

\section{3. ЭКВИВАЛЕНТНЫЕ НАПРЯЖЕНИЯ}

На рис. 4 и 5 показано распределение эквивалентных (по Мизесу) напряжений в лицевой части верхнечелюстного комплекса и в основании черепа соответственно для двух различных конструкций 
ортодонтического аппарата. Случай а соответствует конструкции аппарата с горизонтальным расположением винта и стержней, случай $б-$ конструкции, в которой винт смещен на 8 мм относительно горизонтальной положения аппарата ближе к небу.

Из распределений напряжений, показанных на рис. 4, a и 5, а видно, что значительные напряжения возникают, в основном, в области верхней челюсти; максимальные эквивалентные напряжения составляют $\approx 74.3$ МПа. Достаточно высокие напряжения наблюдаются в средней и нижней части носового хода. Присутствуют напряжения в нижней области левой глазницы. То обстоятельство, что напряженное состояние испытывает только одна глазница можно объяснить асимметрией верхнечелюстного комплекса. Этим также обусловлено возникновение более высоких напряжений в области левого подглазничного отверстия по сравнению с правым (33.0 и 16.5 МПа соответственно).

Из рис. 4, б видно, что в случае установки винта аппарата у неба, верхняя челюсть нагружается фрагментарно, и наибольшие эквивалентные напряжения равны 20.12 МПа. В частности, напряжения наблюдаются в области альвеолярных и лобных отростков верхней челюсти, а также в области лобноносового шва. Также следует отметить возникновение незначительных напряжений в клиновидной и затылочной костях.

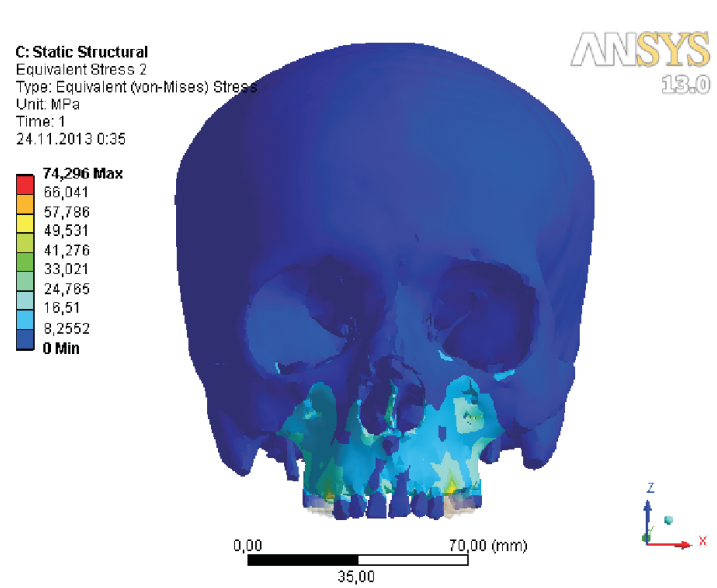

a

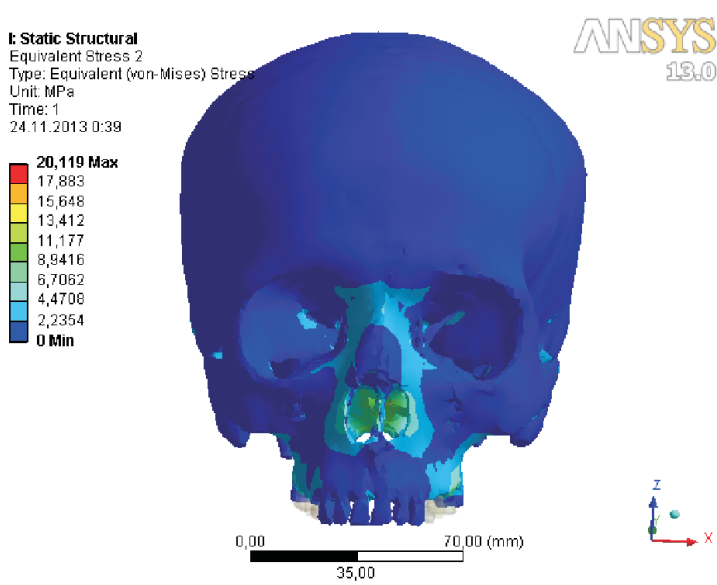

б

Рис. 4. Распределение эквивалентных напряжений в лицевой части черепа после активации ортодонтического аппарата (значения напряжений приведены в МПа): $a-$ стержни и пластинки отодонтического аппарата расположены в горизонтальной плоскости; б - пластинки ортодонтического аппарата смещены к верхнему небу на 8 мм по отношению к горизонтальному положению

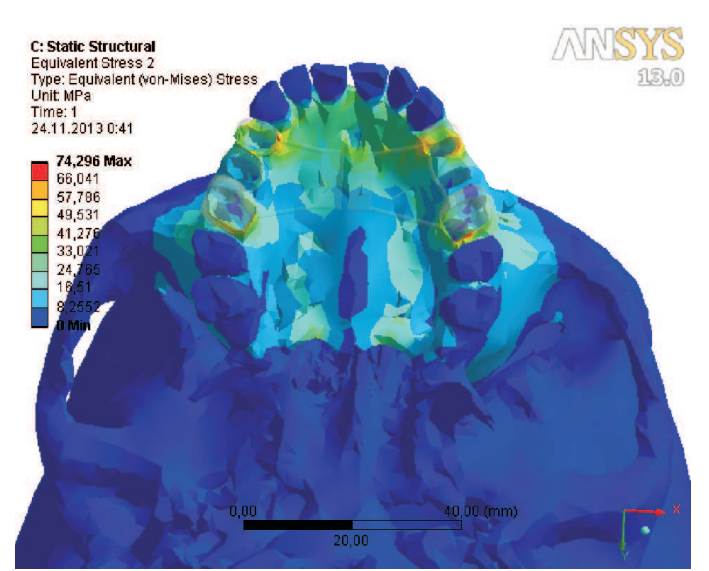

$a$

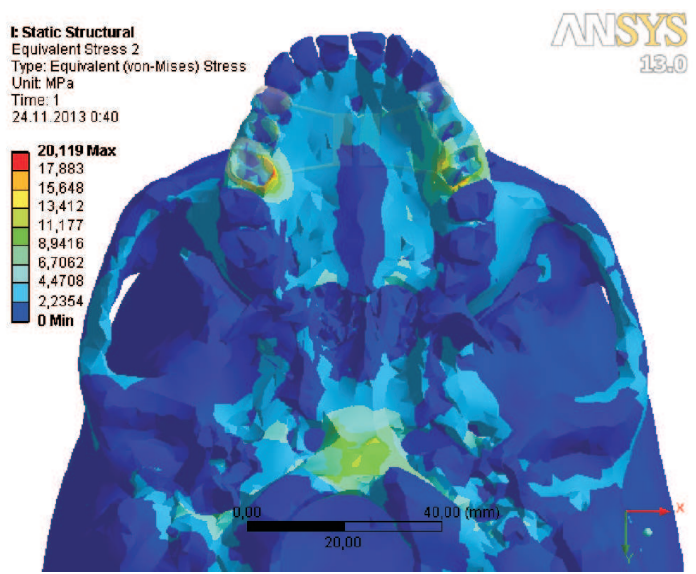

$\sigma$

Рис. 5. Распределение эквивалентных напряжений в основании черепа после активации ортодонтического аппарата (значения напряжений приведены в МПа): $a-$ стержни и пластинки отодонтического аппарата расположены в горизонтальной плоскости; б - пластинки ортодонтического аппарата смещены к верхнему небу на 8 мм по отношению к горизонтальному положению 
Как показывает рис. 5, б, практически не нагружается срединный небный шов, посредством раскрытия которого, как правило, осуществляется расширение верхней челюсти. Поэтому случай $a$ установки ортодонтического аппарата более предпочтителен, чем вариант б, для быстрого раскрытия срединного небного шва. Однако следует учитывать, что при горизонтальной установке ортодонтического аппарата будут возникать значительные, по сравнению со случаем б, ортодонтические силы, действие которых при активации винта может сопровождаться сильными болевыми ощущениями.

Другим важным обстоятельством является то, что наибольшие напряжения, как случае $a$, так и в случае б, возникают в костной ткани, окружающей зубы, на которые устанавливается ортодонтический аппарат. Это обстоятельство подтверждают диаграммы распределения эквивалентных напряжений в основании черепа, показанные на рис. $5, a$, б. Значения напряжений в костной ткани, с которой контактируют опорные зубы, находятся в диапазоне от 30.02 до 74.30 МПа (случай $a$ ) и от 8.94 до 20.12 МПа (случай б). В то же время кости черепа по-разному нагружаются в случаях $a$ и 6 установки ортодонтического аппарата. Так, в случае $a$ высокие напряжения возникают в резцовой кости (33.02 МПа), верхней челюсти и небной кости $(8.26$ - $33.02 \mathrm{MПа).} \mathrm{В} \mathrm{случае} \mathrm{б} \mathrm{напряжения} \mathrm{в}$ верхней челюсти (6.71 МПа) меньше напряжений, возникающих в затылочной кости, в частности в области большого затылочного отверстия (11.18 МПа). Кроме того, в случае б ненулевые напряжения возникают в скуловых дугах, а также в крыловидной пластинке.

\section{4. ПЕРЕМЕЩЕНИЯ ТОЧЕК ЧЕРЕПА И ОПОРНЫХ ЗУБОВ}

Векторные поля суммарных перемещений точек верхнечелюстного комплекса и опорных зубов для случаев $a$ и $\sigma$ установки ортодонтического аппарата показаны на рис. 6 и 7 соответственно.

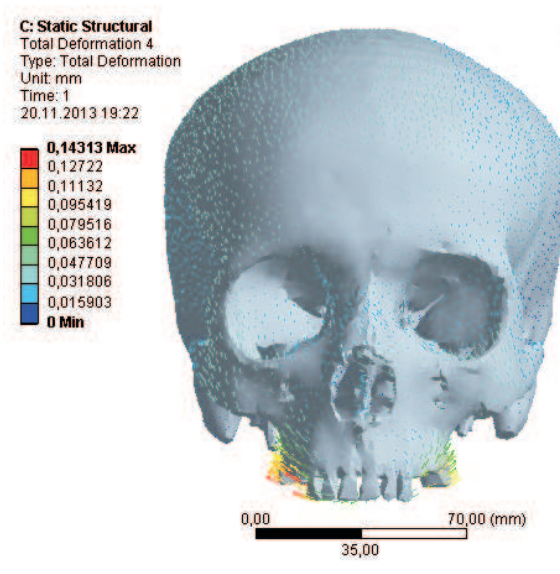

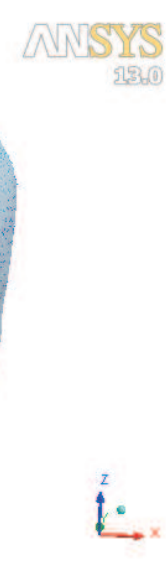

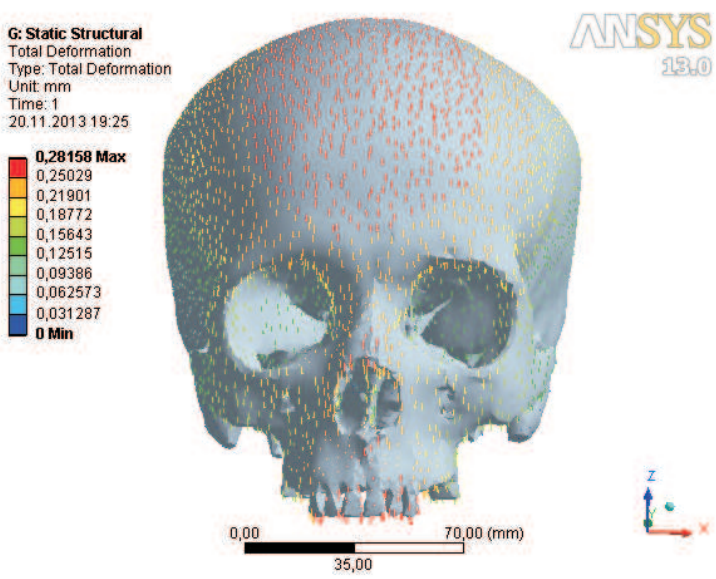

б

Рис. 6. Полные перемещения точек черепа (значения перемещений приведены в миллиметрах) после активации ортодонтического аппарата: $a-$ стержни и пластинки отодонтического аппарата расположены в горизонтальной плоскости; б - пластинки ортодонтического аппарата смещены к верхнему небу на 8 мм по отношению к горизонтальному положению

Видно, что направление перемещений существенно изменяется при установке пластинок ортодонтического аппарата у верхнего неба (см. рис. 6,6$)$ по сравнению с направлениями перемещений при горизонтальном положении стержней и пластинок аппарата (см. рис. 6, a). В случае $a$ составляющая вектора полных перемещений верхней челюсти, коллинеарная оси аппликат (оси $0 z$ ), направлена вдоль положительного направлени этой оси. В случае б вектора суммарных перемещений (в том числе и составляющая коллинеарная оси аппликат) направлены вдоль отрицательного направления оси $0 z$. В случае $a$ наибольшие суммарные перемещения наблюдаются для точек верхней челюсти и составляют 0.1432 мм. Суммарные перемещения других костей черепа существенно меньше, в частности, наибольшие перемещения скуловой кости $\approx 0.048$ мм; для остальных костей черепа величины перемещений не превышают этого значения. В случае $\sigma$ наибольшие перемещения составляют $\approx 0.2816$ мм и наблюдаются у большинства костей лицевой части черепа, в частности, у верхней челюсти, носовой кости, лобной кости и других костей. Наибольшие суммарные перемещения в случае $\sigma$ по сравнению со случаем $a$ увеличиваются почти в два раза. 

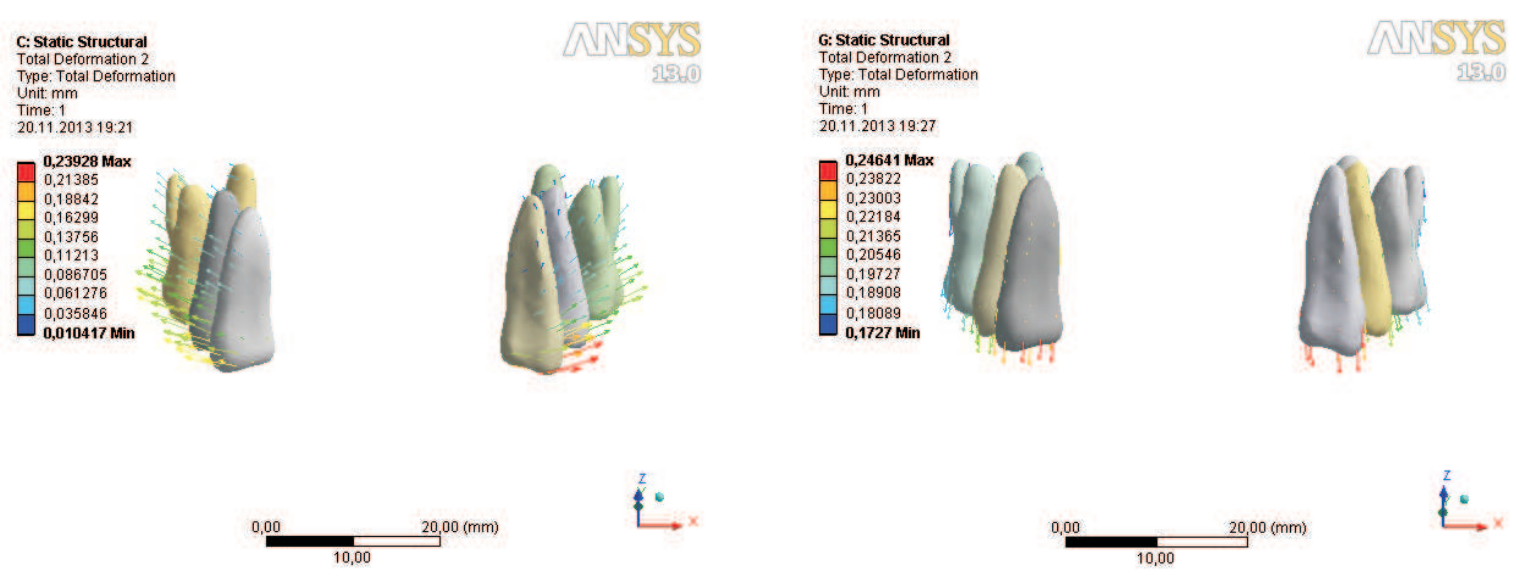

$\stackrel{2}{\longrightarrow}$

a
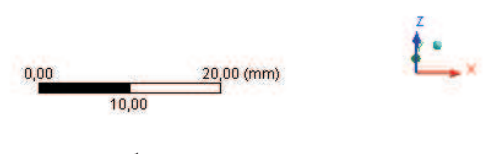

б

Рис. 7. Полные перемещения опорных зубов верхней челюсти (значения перемещений приведены в миллиметрах) после активации ортодонтического аппарата: $a$ - стержни и пластинки отодонтического аппарата расположены в горизонтальной плоскости; б - пластинки ортодонтического аппарата смещены к верхнему небу на 8 мм по отношению к горизонтальному положению

Полные перемещения опорных зубов в случае $a$ установки ортодонтического аппарата, так же как и полные перемещения точек верхней челюсти, в основном направлены латерально и вверх (см. рис. $7, a$ ). Наблюдаемое направление перемещений указывает на наклонно-вращательное смещение зубов верхнего зубного ряда. Кроме того, направления полных перемещений премоляра левой стороны (см. рис. $7, a$ ) таковы, что зуб поворачивается относительно центра вращения, находящегося на самом зубе, что может привести к выворачиванию зуба в ходе ортодонтического лечения. Сравнение рис. $6, a$ и $7, a$ показывает, что значения наибольших перемещений опорных зубов превышают величины максимальных перемещений точек черепа. Это объясняется тем, что центры вращения зубов, на которые опирается ортодонтический аппарат, находятся на корне зуба или на черепе. Поворачиваясь относительно своего центра вращения, зуб получает перемещения, которые увеличиваются при удалении от центра вращения. Перемещения внешней поверхности корня зуба и внутренней поверхности зубной альвеолы совпадают. Указанное отличие перемещений зубов и черепа при горизонтальном расположении ортодонтического аппарата можно трактовать таким образом, что на опорные зубы оказывается более значительное воздействие, чем на кости черепа.

В случае установки ортодонтического аппарата у неба полные перемещения практически полностью направлены вниз (см. рис. 7,6 ). В отличие от случая $a$, максимальное значение перемещений опорных зубов меньше, чем наибольшее значение полных перемещений точек верхнечелюстного комплекса. Учитывая это обстоятельство, а также направление перемещений зубов и черепа, показанные на рис. 6,6 и 7,6 соответственно, можно сделать вывод, что зубы перемещаются за счет перемещения всего верхнечелюстного комплекса. Аналогичное векторное поле для полных перемещений наблюдается при конструкциях ортодонтического аппарата с расположением винта на 0.5, 1 и 2 мм выше по сравнению с горизонтальной плоскостью, в которой находится винт и стержни аппарата в случае $a$. В большинстве конечно-элементных исследований указывается на вращение верхнечелюстного комплекса в сагиттальной плоскости $[2,23]$, а также на незначительную экструзию нагружаемых зубов [2], что согласуется с полученными результатами для перемещений.

Отметим, что в ходе конечно-элементного анализа напряженно-деформированного верхнечелюстного комплекса при активации ортодонтического аппарата исследование сеточной сходимости выполнялось для черепа с горизонтальным расположением аппарата следующим образом. На первом этапе проведен конечно-элементный расчет при автоматическом конечно-элементном разбиении (принятым по умолчанию в пакете ANSYS Workbench) с количеством узлов и элементов, указанным в статье. На втором и третьем этапах выполнен конечно-элементный расчет черепа с аппаратом при измененной вычислительной сетке для черепа с количеством узлов, увеличенным в два и четыре раза соответственно. Увеличение узлов для модели ортодонтического аппарата не выполнялось, поскольку работа не была направлена на конечно-элементный анализ напряженно-деформированного состояния аппарата. Изменение вычислительной сетки осуществлялось в пакете MIMICS. Сравнительный анализ полученных результатов расчета проводился для определенной области черепа, охватывающей 
часть верхней челюсти и неба. Анализ показал, что значения перемещений не изменяются; значения эквивалентных напряжений практически не изменяются, в частности максимальное значение эквивалентных напряжений в рассматриваемой области на первом, втором и третьем этапах составило $\approx 59.98$ МПа, $\approx 60,01$ МПа и $\approx 60,012$ МПа соответственно. Поэтому, чтобы избежать многократного увеличения времени расчета модели при мелкой сетке, расчеты проводились при автоматическом режиме конечно-элементного разбиения.

\section{ЗАКЛЮЧЕНИЕ}

Подход к конечно-элементному исследованию поведения верхнечелюстного комплекса при верхнечелюстном расширении, основанный на приложении нагрузки непосредственно от ортодонтического аппарата, позволяет более полно и точно смоделировать действие ортодонтических сил, объяснить клинически наблюдаемые последствия [6-10] и систематизировать результаты вычислительных экспериментов [18-31].

Расчет напряженно-деформированного состояния верхнечелюстного комплекса при активации ортодонтического аппарата с винтом HYRAX показал, что конструкции с винтом, расположенным в одной горизонтальной плоскости со стержнями, и винтом, расположенным у неба, не являются оптимальными. В первом случае возникают необходимые напряжения в области срединного небного шва, но появляется существенное наклонно-вращательное перемещение опорных зубов и латеральноверхнее смещение верхней челюсти. Во втором случае, а также для случаев расположения винта аппарата на 0.5, 1 и 2 мм выше горизонтального расположения аппарата, в области срединного небного шва возникают незначительные напряжения и, наоборот, напряжения испытывают те области черепа, воздействие на которые нежелательно. Анализ зависимостей эквивалентных напряжений и компонент полных перемещений, направленных вдоль координатных осей, от расстояния винта до окклюзионной плоскости для черепа и опорных зубов показал, что трансверсальное перемещение верхней челюсти наблюдается в случаях, когда винт ортодонтического аппарата расположен на расстоянии 0.20-0.22 мм от горизонтального положения ближе к небу. При таком расположении возникают достаточно высокие напряжения в верхней челюсти и в области срединного небного шва и практически отсутствуют вертикальные перемещения опорных зубов и черепа.

Работа выполнена в рамках Государственной программы научных исследований «Конвергенцзия» (Задание 1.8.01).

\section{Библиографрический список}

1. Chaconas S. J., Caputo A. A. Observation of orthopedic force distribution produced by maxillary orthodontic appliances // Am. J. Orthod. 1982. Vol. 82. P. 492-501. 2. Iseri H., Tekkaya A. E., Oztan O., Bilgic S. Biomechanical effects of rapid maxillary expansion on the craniofacial skeleton, studied by the finite element method // Eur. J. Orthod. 1998. Vol. 20. P. 347-356. 3. Provatidis C., Georgiopoulos B., Kotinas A., McDonald $J$. $P$. On the FEM modeling of craniofacial changes during rapid maxillary expansion // Med. Eng. Phys. 2007. Vol. 29. P. 566-579.

4. Wang D., Cheng L., Wang C., Qian Y., Pan X. Biomechanical analysis of rapid maxillary expansion in the UCLP patient // Med. Eng. Phys. 2009. Vol. 31. P. 409-417.

5. Ludwig B., Baumgaertel S., Zorkun B., Bonitz L., Glasl B., Wilmes B., Lisson J. Application of a new viscoelastic finite element method model and analysis of miniscrew-supported hybrid hyrax treatment // Am. J. Orthod. Dentofac. Orthop. 2013. Vol. 143. P. 426-435.

6. Chung C. H., Font B. Skeletal and dental changes in the sagittal, vertical, and transverse dimensions after rapid palatal expansion // Am. J. Orthod. Dentofac. Orthop. 2004. Vol. 126. P. 569-575.

7. Cozzani M., Rosa M., Cozzani P., Siciliani G. Deciduous dentitionanchored rapid maxillary expansion in crossbite and non-crossbite mixed dentition patients: reaction of the permanent first molar // Prog. Orthod. 2003. Vol. 4. P. 15-22.

8. Wertz R. A. Skeletal and dental changes accompanying rapid midpalatal suture opening // Am. J. Orthod. 1970. Vol. 58. P. 41-46.

9. Timms D. J. A study of basal movement with rapid maxillary expansion // Am. J. Orthod. 1980. Vol. 77. P. 500-507.

10. Baccetti T., Franchi L., Cameron C. G., McNamara J. A. Jr. Treatment timing for rapid maxillary expansion // Angle Orthod. 2001. Vol. 71. P. 343-350.

11. Shetty V., Caridad J. M., Caputo A. A., Chaconas $S$. J. Biomechanical rationale for surgicalorthodontic expansion of the adult maxilla // J. Oral Maxillofac. Surg. 1994. Vol. 52. P. 742-749.

12. Pavlin D., Vukicevic D. Mechanical reactions of facial skeleton to maxillary expansion determined by laser holography // Am. J. Orthod. 1984. Vol. 85. P. 498-507. 13. Kragt G., Duterloo H. S., Ten Bosch J. J. The initial reaction of a macerated human skull caused 
by orthodontic cervical traction determined by laser metrology // Am. J. Orthod. 1982. Vol. 81. P. 49-56.

14. Braun S., Bottrel J. A., Lee K. G., Lunazzi J. J., Legan H. L. The biomechanics of maxillary sutural expansion // Am. J. Orthod. Dentofac. Orthop. 2000. Vol. 118. P. 257-261.

15. Book D., Lavelle C. Changes in craniofacial size and shape with two modes of orthodontic treatment // J. Craniofac. Genet. Dev. Biol. 1988. Vol. 8. P. 207-223.

16. Tanne K., Miyasaka J., Yamagata Y., Sakuda M., Burstone C. J. Biomechanical changes in the craniofacial skeleton by the rapid expansion appliance // J. Osaka Univ. Dental Soc. 1985. Vol. 30. P. 345-356.

17. Nakagawa M., Ichikawa K. Biomechanical effects of maxillary protraction on the craniofacial complex on the strain gauge measurements // J. Japan Orthod. Soc. 1986. Vol. 45. P. 109-118.

18. Boryor A., Geigera M., Hohmann A., Wunderlich A., Sander C., Sander F. M., Sander F. G. Stress distribution and displacement analysis during an intermaxillary disjunction A three-dimensional FEM study of a human skull // J. Biomech. 2008. Vol. 41. P. 376-382.

19. Capelozza Filho L., De Almeida A. M., Ursi W. J. Rapid maxillary expansion in cleft lip and palate patients // J. Clin. Orthod. 1994. Vol. 28. P. 34-39.

20. Cattaneo P., Dalstra M., Melsen B. The transfer of occlusal forces through the maxillary molars: a finite element study // Am. J. Orthod. Dentofac. Orthop. 2003. Vol. 123. P. 367-373.

21. Gautam P., Valiathan A., Adhikari R. Stress and displacement patterns in the craniofacial skeleton with rapid maxillary expansion: a finite element method study // Am. J. Orthod. Dentofac. Orthop. 2007. Vol. 132. P. 5.e1-5.e11.

22. Holberg C., Holberg N., Schwenzer K., Wichelhaus A., Rudzki-Janson I. Biomechanical analysis of maxillary expansion in CLP patients // Angle Orthod. 2007. Vol. 77. P. 280-287.

23. Jafari A., Shetty K. S., Kumar M. Study of stress distribution and displacement of various craniofacial structures following application of transverse orthopedic forces-a three dimensional FEM study // Angle Orthod. 2003. Vol. 73. P. 12-20.

24. Lee H., Ting K., Nelson M., Sun N., Sung S. J. Maxillary expansion in customized finite element method models // Am. J. Orthod. Dentofac. Orthop. 2009 Vol. 136. P. 367-374.

25. Miyasaka-Hiraga J., Tanne K., Nakamura S. Finite element analysis for stresses in the craniofacial sutures produced by maxillary protraction forces applied at the upper canines // Br. J. Orthod. 1994. Vol. 21. P. 343348 .

26. Nicholson P. T., Plint D. A. A long-term study of rapid maxillary expansion and bone grafting in cleft lip and palate patients // Eur. J. Orthod. 1989. Vol. 11. P. 186-192.

27. Pan X., Qian Y., Yu J., Wang D., Tang Y., Shen G. Biomechanical effects of rapid palatal expansion on the craniofacial skeleton with cleft palate: a three-dimensional finite element analysis // Cleft Palate Craniofac J. 2007. Vol. 44. P. 149-154.

28. Tanne K., Hiraga J., Kakiuchi K., Yamagata Y., Sakuda M. Biomechanical effect of anteriorly directed extraoral forces on the craniofacial complex: a study using the finite element method // Am. J. Orthod. Dentofac. Orthop. 1989. Vol. 95. P. 200-207.

29. Tindlund R. S., Rygh P., Boe O. E. Intercanine widening and sagittal effect of maxillary transverse expansion in patients with cleft lip and palate during the deciduous and mixed dentitions // Cleft Palate Craniofac. J. 1993. Vol. 30. P. 195-207.

30. Yu H. S., Baik H. S., Sung S. J., Kim K. D., Cho Y. S. Three-dimensional finite-element analysis of maxillary protraction with and without rapid palatal expansion // Eur. J. Orthod. 2007. Vol. 29. P. 118-125.

31. Landes C. A., Laudermann K., Petruchin O., Mack M. G., Kopp S., Ludwig B., Sader R. A., Seitz O. Comparison of bipartite versus tripartite osteotomy for maxillary transversal expansion using 3-dimensional preoperative and postexpansion computed tomography data // J. Oral. Maxillofac. Surg. 2009. Vol. 67. P. 22872301.

32. Zimring J. F., Isaacson R. J. Forces produced by rapid maxillary expansion III. Forces present during retention // Angle Orthod. 1965. Vol. 35. P. 178-186. 33. Wood S. A., Strait D. S., Dumont E. R., Ross C. F., Grosse I. R. The effects of modeling simplifications on craniofacial finite element models: The alveoli (tooth sockets) and periodontal ligaments // J. Biomech. 2011. Vol. 44. P. 1831-1838.

\section{Finite Element Analysis of the Influence of the Orthodontic Appliance Design on the Maxillary Expansion}

\section{S. M. Bosiakov ${ }^{1}$, A. N. Dosta ${ }^{2}$, A. V. Vinokurova ${ }^{1}$}

\footnotetext{
${ }^{1}$ Belarusian State University, Belarus, 220030, Minsk, Nezavisimosti ave., 4, bosiakov@ bsu.by, janeraven@mail.ru

2 Belarusian State Medical University, Belarus, 220116, Minsk, Dzerjinskogo ave., 83, dostastom75@ mail.ru
}

In present paper the results of the stress-strain state finite element analysis of the human maxillary complex after activating orthodontic appliance are performed. Skull and abutment teeth models are obtained on the basis of the tomographic data of the dry intact adult skull. Orthodontic appliance designs are differ in the arrangement of rods and screws relative to the sky. The equivalent stresses and displacements of the maxillary bones and supporting the teeth are evaluated. It is shown that the horizontal location of orthodontic appliance screw and rods in the skull bones having the highest stresses, but there tipping teeth and upward movement of the maxillary 
bones are observed. For orthodontic appliance activating with a screw located near the palate, there are decreased stresses on the maxillary bone complex, and bones and supporting teeth are moved down. The positions screw orthodontic appliance to translation the upper jaw bones are assessed.

Key words: rapid maxillary expansion, orthodontic device HYRAX, finite-element analysis, stress-strain state, craniofacial complex.

\section{References}

1. Chaconas S. J., Caputo A. A. Observation of orthopedic force distribution produced by maxillary orthodontic appliances. Am. J. Orthod., 1982, vol. 82. pp. 492-501.

2. Iseri H., Tekkaya A. E., Oztan O., Bilgic S. Biomechanical effects of rapid maxillary expansion on the craniofacial skeleton, studied by the finite element method. Eur. J. Orthod., 1998, vol. 20, pp. 347-356.

3. Provatidis C., Georgiopoulos B., Kotinas A., McDonald J. P. On the FEM modeling of craniofacial changes during rapid maxillary expansion. Med. Eng. Phys., 2007, vol. 29. pp. 566-579.

4. Wang D., Cheng L., Wang C., Qian Y., Pan X. Biomechanical analysis of rapid maxillary expansion in the UCLP patient. Med. Eng. Phys., 2009, vol. 31. pp. $409-417$.

5. Ludwig B., Baumgaertel S., Zorkun B., Bonitz L., Glasl B., Wilmes B., Lisson J. Application of a new viscoelastic finite element method model and analysis of miniscrew-supported hybrid hyrax treatment. Am. J. Orthod. Dentofac. Orthop., 2013, vol. 143. pp. 426-435. 6. Chung C. H., Font B. Skeletal and dental changes in the sagittal, vertical, and transverse dimensions after rapid palatal expansion. Am. J. Orthod. Dentofac. Orthop., 2004, vol. 126, pp. 569-575.

7. Cozzani M., Rosa M., Cozzani P., Siciliani G. Deciduous dentitionanchored rapid maxillary expansion in crossbite and non-crossbite mixed dentition patients: reaction of the permanent first molar. Prog. Orthod., 2003, vol. 4. pp. 15-22.

8. Wertz R. A. Skeletal and dental changes accompanying rapid midpalatal suture opening. Am. J. Orthod., 1970, vol. 58 , pp. 41-46.

9. Timms D. J. A study of basal movement with rapid maxillary expansion. Am. J. Orthod., 1980, vol. 77, pp. 500-507.

10. Baccetti T., Franchi L., Cameron C. G., McNamara J. A. Jr. Treatment timing for rapid maxillary expansion. Angle Orthod., 2001, vol. 71, pp. 343-350.

11. Shetty V., Caridad J. M., Caputo A. A., Chaconas S. J. Biomechanical rationale for surgical-orthodontic expansion of the adult maxilla. J. Oral Maxillofac. Surg., 1994, vol. 52, pp. 742-749.

12. Pavlin D., Vukicevic D. Mechanical reactions of facial skeleton to maxillary expansion determined by laser holography. Am. J. Orthod., 1984, vol. 85, pp. 498-507. 13. Kragt G., Duterloo H. S., Ten Bosch J. J. The initial reaction of a macerated human skull caused by orthodontic cervical traction determined by laser metrology. Am. J. Orthod., 1982, vol. 81. pp. 49-56.

14. Braun S., Bottrel J. A., Lee K. G., Lunazzi J. J., Legan H. L. The biomechanics of maxillary sutural expansion. Am. J. Orthod. Dentofac. Orthop., 2000, vol. 118. pp. 257-261.

15. Book D., Lavelle C. Changes in craniofacial size and shape with two modes of orthodontic treatment. $J$.
Craniofac. Genet. Dev. Biol., 1988, vol. 8, pp. 207-223.

16. Tanne K., Miyasaka J., Yamagata Y., Sakuda M., Burstone C. J. Biomechanical changes in the craniofacial skeleton by the rapid expansion appliance. J. Osaka Univ. Dental Soc., 1985, vol. 30, pp. 345-356.

17. Nakagawa M., Ichikawa K. Biomechanical effects of maxillary protraction on the craniofacial complex on the strain gauge measurements. J. Japan Orthod. Soc., 1986, vol. 45, pp. 109-118.

18. Boryor A., Geigera M., Hohmann A., Wunderlich A., Sander C., Sander F. M., Sander F. G. Stress distribution and displacement analysis during an intermaxillary disjunction - A three-dimensional FEM study of a human skull. J. Biomech., 2008, vol. 41, pp. 376-382.

19. Capelozza Filho L., De Almeida A. M., Ursi W. J. Rapid maxillary expansion in cleft lip and palate patients. J. Clin. Orthod., 1994, vol. 28, pp. 34-39.

20. Cattaneo P., Dalstra M., Melsen B. The transfer of occlusal forces through the maxillary molars: a finite element study. Am. J. Orthod. Dentofac. Orthop., 2003, vol. 123, pp. 367-373.

21. Gautam P., Valiathan A., Adhikari R. Stress and displacement patterns in the craniofacial skeleton with rapid maxillary expansion: a finite element method study. Am. J. Orthod. Dentofac. Orthop., 2007, vol. 132, pp. 5.e1-5.e11.

22. Holberg C., Holberg N., Schwenzer K., Wichelhaus A., Rudzki-Janson I. Biomechanical analysis of maxillary expansion in CLP patients. Angle Orthod., 2007, vol. 77, pp. 280-287.

23. Jafari A., Shetty K. S., Kumar M. Study of stress distribution and displacement of various craniofacial structures following application of transverse orthopedic forces-a three dimensional FEM study. Angle Orthod., 2003, vol. 73, pp. 12-20.

24. Lee H., Ting K., Nelson M., Sun N., Sung S. J. Maxillary expansion in customized finite element method models. Am. J. Orthod. Dentofac. Orthop., 2009, vol. 136, pp. 367-374.

25. Miyasaka-Hiraga J., Tanne K., Nakamura S. Finite element analysis for stresses in the craniofacial sutures produced by maxillary protraction forces applied at the upper canines. Br. J. Orthod., 1994, vol. 21, pp. 343-348. 26. Nicholson P. T., Plint D. A. A long-term study of rapid maxillary expansion and bone grafting in cleft lip and palate patients. Eur. J. Orthod., 1989, vol. 11, pp. 186192.

27. Pan X., Qian Y., Yu J., Wang D., Tang Y., Shen G. Biomechanical effects of rapid palatal expansion on the craniofacial skeleton with cleft palate: a three-dimensional finite element analysis. Cleft Palate Craniofac J., 2007, vol. 44, pp. 149-154.

28. Tanne K., Hiraga J., Kakiuchi K., Yamagata Y., Sakuda M. Biomechanical effect of anteriorly directed extraoral forces on the craniofacial complex: a study 
using the finite element method Am. J. Orthod. Dentofac. Orthop., 1989, vol. 95, pp. 200-207.

29. Tindlund R. S., Rygh P., Boe O. E. Intercanine widening and sagittal effect of maxillary transverse expansion in patients with cleft lip and palate during the deciduous and mixed dentitions. Cleft Palate Craniofac. J., 1993, vol. 30, pp. 195-207.

30. Yu H. S., Baik H. S., Sung S. J., Kim K. D., Cho Y. S. Three-dimensional finite-element analysis of maxillary protraction with and without rapid palatal expansion. Eur. J. Orthod., 2007, vol. 29, pp. 118-125.

31. Landes C. A., Laudermann K., Petruchin O., Mack M. G., Kopp S., Ludwig B., Sader R. A., Seitz O.
Comparison of bipartite versus tripartite osteotomy for maxillary transversal expansion using 3-dimensional preoperative and postexpansion computed tomography data. J. Oral. Maxillofac. Surg., 2009, vol. 67, pp. 22872301.

32. Zimring J. F., Isaacson R. J. Forces produced by rapid maxillary expansion III. Forces present during retention. Angle Orthod., 1965, vol. 35, pp. 178-186.

33. Wood S. A., Strait D. S., Dumont E. R., Ross C. F., Grosse I. R. The effects of modeling simplifications on craniofacial finite element models: The alveoli (tooth sockets) and periodontal ligaments. J. Biomech., 2011, vol. 44, pp. 1831-1838.

УДК 539.3

\title{
ОДНОМЕРНАЯ ЗАДАЧА О НЕСТАЦИОНАРНОЙ СВЯЗАННОЙ УПРУГОЙ ДИФФУЗИИ ДЛЯ СЛОЯ
}

\begin{abstract}
А. Р. Гачкевич ${ }^{1}$, А. В. Земсков ${ }^{2}$, Д. В. Тарлаковский ${ }^{3}$
${ }^{1}$ Доктор фозико-математических наук, просрессор, Институт прикладных проблем механики и математики им. Я. С. Подстригача НАН Украины, Львов, dept13@iapmm.Iviv.ua

${ }^{2}$ Кандидат фризико-математических наук, доцент кафедры математического моделирования, Московский авиационный институт (национальный исследовательский университет), azemskov1975@mail.ru

${ }^{3}$ Доктор фризико-математических наук, заведующий кафедрой сопротивления материалов и деталей машин, профрессор, Московский авиационный институт (национальный исследовательский университет), tdvhome@mail.ru

Рассматривается задача об определении напряжённо-десрормированного состояния упругой среды с учётом структурных изменений, обусловленных наличием диффрузионных потоков. Влияние дифффузионных процессов на напряжённодесрормированное состояние среды учитывается с помощью локально равновесной модели упругой дифффузии, включающей в себя связанную систему уравнений движения упругого тела и уравнения массопереноса. Для решения используется разложение искомых фрункций в ряды Фурье с последующим применением интегрального преобразования Лапласа по времени. Строится фрундаментальное решение задачи. Рассмотрены примеры для случаев, когда дисрфузионный поток на границе постоянен или затухает по экспоненциальному закону.
\end{abstract}

Ключевые слова: упругая дифрфузия, нестационарные задачи, ряды Фурье, преобразование Лапласа.

\section{ВВЕДЕНИЕ}

Во многих случаях необходимо учитывать эффекты связанности полей различной природы с целью получения, например, более точного описания воздействия статических и динамических нагрузок и диффузии на упругие среды и элементы современных конструкций, в том числе объектов авиационной и ракетно-космической техники. При этом переменное по времени поле деформаций инициирует диффузионные потоки, наличие которых приводит к перераспределению компонентов вещества. Поэтому в диффузионной зоне возникает напряжённо-деформированное состояние, которое, в свою очередь, влияет на величину диффузионного потока.

Соотношения между параметрами, характеризующими такую систему, строятся с помощью метода потенциалов, разработанного Дж. Гиббсом. При этом в качестве потенциалов выступают внутренняя энергия, свободная энергия и химический потенциал (или удельный потенциал Гиббса). Основополагающими законами, позволяющими выразить термодинамические потенциалы через термодинамические параметры, являются первый и второй законы термодинамики. Для вывода уравнений, описывающих физико-механические процессы в деформируемой среде, применяются уравнения движения упругого тела и законы массопереноса [1-4]. При этом используются следующие предположения:

1) изменения концентрации, сопутствующие деформированию, малы;

2) возрастание концентрации не приводит к существенным изменениям упругих и диффузионных характеристик материала, поэтому эти константы рассматриваются как величины, не зависящие от концентраций;

3) деформации малы. 